\title{
Non-cardiac, non-oesophageal chest pain: the relevance of psychological factors
}

\author{
K Y Ho, J Y Kang, B Yeo, W L Ng
}

\begin{abstract}
Background-No cause has been determined for chest pain that is neither cardiac nor oesophageal in origin.

Aims-To compare the prevalence of lifetime psychiatric disorders and current psychological distress in three consecutive series of patients with chronic chest or abdominal pain.

Patients-Thirty nine patients with noncardiac chest pain and no abnormality on oesophagogastroduodenoscopy, oesophageal manometry, and 24 hour pH monitoring; 22 patients with non-cardiac chest pain having endoscopic abnormality, oesophageal dysmotility, and/or pathological reflux; and 36 patients with biliary colic.

Methods-The Diagnostic Interview Schedule and the 28 item General Health Questionnaire were administered to all patients.
\end{abstract}

Results-Patients with non-cardiac chest pain and no upper gastrointestinal disease had a higher proportion of panic disorder $(15 \%)$, obsessive-compulsive disorder $(21 \%)$, and major depressive episodes $(28 \%)$ than patients with gallstone disease $(0 \%, p<0.02 ; 3 \%, p<0.02$; and $8 \%, p<0.05$, respectively). In contrast, there were no differences between patients with noncardiac chest pain and upper gastrointestinal disease and patients with gallstone disease in any of the DSM-111 defined lifetime psychiatric diagnoses. Using the General Health Questionnaire, $49 \%$ of patients with non-cardiac chest pain without upper gastrointestinal disease scored above the cut off point (that is, more than 4), which was considered indicative of non-psychotic psychiatric disturbance, whereas only $14 \%$ of patients with gallstones did so $(p<0.005)$. The proportions of such cases were however similar between patients with non-cardiac chest pain and upper gastrointestinal disease $(27 \%)$ and patients with gallstones.

Conclusions-Psychological factors may play a role in the pathogenesis of chest pain that is neither cardiac nor oesophagogastric in origin.

(Gut 1998;43:105-110)

Keywords: chest pain; oesophageal manometry; gastro-oesophageal reflux disease; oesophageal $\mathrm{pH}$ monitoring; psychiatric illness

Dr K Y Ho, Department of

Medicine, National

University Hospital, Lower

Kent Ridge Road, Singapore

119074

Accepted for publication 4 February 1998
Recurrent chest pain of non-cardiac origin is a frequent clinical problem. We and others have previously shown that gastro-oesophageal re- flux disease is the most common cause of such pain, accounting for approximately one third of cases. ${ }^{1-3}$ No cause has been determined for chest pain that is neither cardiac nor oesophageal in origin. Psychological factors have been suggested as relevant and treatment of underlying psychiatric disorders may improve the patient's chest pain. ${ }^{4}$

Among patients with chest pain, those with normal coronary arteries are more likely to have psychiatric disorders than those with coronary artery disease. ${ }^{5}$ A high prevalence of psychiatric illness has also been noted among subjects with non-specific oesophageal motility disorder and nutcracker oesophagus. ${ }^{67}$ However, it is unclear whether the association between psychological disorders and noncardiac chest pain is influenced by the presence or absence of structural or reflux related oesophageal disease.

To elucidate this, the present study compared the prevalence of lifetime psychiatric disorders and current psychological distress among three consecutive series of patients with chronic chest or abdominal pain: (1) patients with non-cardiac chest pain and clinically significant upper gastrointestinal disorders; (2) patients with non-cardiac chest pain and no upper gastrointestinal disorders; and (3) patients with recurrent biliary colic.

\section{Methods}

PATIENTS

Consecutive patients referred for non-cardiac chest pain to the gastroenterology service at the National University Hospital, Singapore, were invited to participate in the study. Non-cardiac chest pain was defined as recurrent chest pain of more than three months duration, with either: (1) normal or non-obstructive coronary arteries (less than $50 \%$ narrowing of the luminal diameter); (2) normal dobutamine stress echocardiography; or (3) a negative exercise electrocardiogram and a cardiologist's evaluation that the symptoms were not cardiac in origin. Each participant underwent full medical history, followed by physical examination and oesophageal tests including oesophagogastroduodenoscopy, standard oesophageal manometry, and ambulatory 24 hour oesophageal $\mathrm{pH}$ monitoring. Other tests such as upper abdominal ultrasound investigation were performed if clinically indicated. In addition, psychological assessment using questionnaires was carried out on all participants. Subjects were subsequently assigned to one of two groups: those with abnormal oesophageal tests, and those with normal results.

Controls were drawn from consecutive patients with recurrent biliary colic admitted to 
the hospital for elective cholecystectomy. This group was chosen because their clinical features are similar to those seen in patients with non-cardiac chest pain: chronic, recurrent pain which is not life threatening, and the necessity for diagnostic procedures which are sometimes uncomfortable. They were recruited during the same period of time as the patients with non-cardiac chest pain, and for these controls, only the psychological evaluation was performed. Informed consent was obtained from all respondents, and the study was approved by the hospital ethics committee.

\section{STUDY DESIGN}

Oesophagogastroduodenoscopy

Upper gastrointestinal endoscopy was performed to diagnose reflux oesophagitis and to exclude peptic ulcer disease. This was done by experienced endoscopists using Olympus fibreoptic or video gastroscopes. Endoscopic oesophagitis was defined as erosions extending proximally from the $\mathrm{Z}$ line, whether or not ulceration or stenosis was present. Its severity was graded according to a four point scale: grade 1 , non-confluent erosions; grade 2 , noncircumferential confluent erosions; grade 3, circumferential erosions; and grade 4 , ulceration or stricture. Hiatus hernia was defined as an extension of gastric mucosa above the diaphragm for more than $1.5 \mathrm{~cm}$. Endoscopic gastroduodenitis was defined as the presence of erythema, petechiae, or erosion in the absence of peptic ulcer or malignancy. When present, gastric or duodenal ulcers were at least $0.5 \mathrm{~cm}$ in diameter. As hiatus hernia and endoscopic gastroduodenitis have not been proved to cause symptoms, these were regarded as normal findings for the purposes of this study.

\section{Manometry}

Standard oesophageal manometry was performed using a quadruple lumen polyvinyl catheter (inside diameter of each lumen, 0.8 $\mathrm{mm}$; outside diameter of catheter, $3.2 \mathrm{~mm}$ ) with radially orientated side ports spaced $5 \mathrm{~cm}$ apart (Mui Scientific, Ontario, Canada). The lumens were perfused with distilled water at a rate of $0.6 \mathrm{ml} / \mathrm{min}$ from a low compliance pneumohydraulic capillary infusion system (Model PIP-4, Mui Scientific, Ontario, Canada). The catheter was connected to a PC Polygraf HR (Synectics Medical, Stockholm, Sweden), with data recorded on a computer and analysed with an on line computer program (Polygram version 6.31, Gastrosoft Inc., USA).

After a six hour fast, manometry was performed according to a previously described technique. ${ }^{8}$ Medication known to affect oesophageal contractions, acid secretions, and pain perception was withheld for at least 72 hours before the study. Abnormal manometric recordings were categorised according to previously set criteria, ${ }^{9}$ but modified by values obtained in our motility laboratory from a study of 26 asymptomatic healthy volunteers with a median age of 23 years (range 19-34). All manometric tracings were analysed and classified by one of the authors $(\mathrm{KYH})$ without knowledge of the psychiatric results. For the purpose of this study, only achalasia and diffuse oesophageal spasm were considered functionally significant manometric abnormalities, as it has been shown that only these two manometric patterns were found in symptomatic subjects but not asymptomatic ones. ${ }^{10}$

\section{pH monitoring}

Ambulatory 24 hour oesophageal $\mathrm{pH}$ monitoring was carried out, usually on an outpatient basis after the manometry. A monocrystalline antimony $\mathrm{pH}$ electrode (Synectics Medical, Stockholm, Sweden) was passed nasally, and positioned $5 \mathrm{~cm}$ above the manometrically determined upper border of the lower oesophageal sphincter. A separate silver/silver chloride reference electrode was attached to the skin of the non-dominant shoulder. Both electrodes were connected to a digital data recorder (Digitrapper Mark 11 Gold, Synectics Medical, Stockholm, Sweden), which sampled $\mathrm{pH}$ activity at a rate of $4 \mathrm{~Hz}$ for at least 24 hours. Subjects returned to their homes or work places and resumed normal activities. They were instructed to eat their usual diet, but to avoid drinks and foods with a pH below 4 . Subjects returned to the hospital after 24 hours for removal of the apparatus.

Data were transferred from the recorder to a personal computer and analysed (EsopHogram version 5.60, Gastrosoft Inc., USA). The onset of a reflux episode was defined as a drop in the oesophageal $\mathrm{pH}$ to less than 4 for at least four seconds, and its end as the first rise above 4. In case of a subsequent fall of $\mathrm{pH}$ below 4 within five seconds, both consecutive reflux episodes were interpreted as one single complex. The total percentage of time the $\mathrm{pH}$ was below 4 (oesophageal acid exposure time) was calculated for each patient. Gastrooesophageal reflux was considered to be present when the percentage exceeded $4.0 \%$. This figure was the 95th percentile value obtained from prior studies done in our laboratory on 10 asymptomatic healthy volunteers with a median age of 23 years (range 20-32).

\section{Psychology}

Psychological assessment was based on two well validated and established standardised psychiatric instruments: the Diagnostic Interview Schedule (DIS) ${ }^{11}$ and the 28 item General Health Questionnaire (GHQ). ${ }^{12}$ The DIS is a highly structured interview designed to make lifetime psychiatric diagnoses according to the criteria outlined in the Diagnostic and Statistical Manual of Mental Disorders-111-R (DSM111-R) of the American Psychiatric Association. ${ }^{13}$ Six diagnoses were sought in this investigation: panic disorder, generalised anxiety disorder, phobic disorder, major depressive episode, obsessive-compulsive disorder, and somatisation disorder. These were chosen as being the most common psychiatric conditions associated with unexplained physical symptoms. ${ }^{14}$

The GHQ is a screening questionnaire for the detection of psychiatric morbidity in the 
Table 1 Sociodemographic and clinical characteristics by subject group

\begin{tabular}{|c|c|c|c|}
\hline & Group I $(n=39)$ & Group II $(n=22)$ & Group III $(n=36)$ \\
\hline $\operatorname{Sex}(M / F)$ & $17 / 22$ & $10 / 12$ & $15 / 21$ \\
\hline \multicolumn{4}{|l|}{ Age $(\mathrm{y})$} \\
\hline Median & 47 & 53 & 49 \\
\hline Range & $21-63$ & $23-76$ & $31-81$ \\
\hline \multicolumn{4}{|l|}{ Ethnicity (\%) } \\
\hline Chinese & 28 & 18 & 27 \\
\hline Other & 11 & 4 & 9 \\
\hline \multicolumn{4}{|l|}{ Education } \\
\hline Below secondary & 14 & 7 & 12 \\
\hline Secondary & 15 & 8 & 15 \\
\hline Above secondary & 10 & 7 & 9 \\
\hline \multicolumn{4}{|l|}{ Marital status } \\
\hline Married & 30 & 14 & 29 \\
\hline Single & 5 & 4 & 5 \\
\hline Divorced/widowed & 4 & 4 & 2 \\
\hline \multicolumn{4}{|l|}{ Occupation ${ }^{\star}$} \\
\hline Non-manual & 12 & 13 & 18 \\
\hline Manual & 8 & 0 & 4 \\
\hline Unemploved & 19 & 9 & 14 \\
\hline \multicolumn{4}{|l|}{ Duration of pain (months) $\dagger$} \\
\hline Mean $(95 \% \mathrm{CI})$ & $27.0(19.7-34.3)$ & $14.7(7.2-22.3)$ & $8.2(6.7-9.7)$ \\
\hline \multicolumn{4}{|l|}{ Manometric findings } \\
\hline Normal & 32 & 16 & \\
\hline Non-specific abnormalities & 6 & 5 & \\
\hline Nutcracker oesophagus & 1 & 0 & \\
\hline Diffuse oesophageal spasm & 0 & 1 & \\
\hline
\end{tabular}

form of non-psychotic psychological illness, and has been used successfully in community and non-psychiatric clinical settings in many countries. ${ }^{15}$ It contains four subscales: somatic symptoms, anxiety and insomnia, social dysfunction, and severe depression; these are termed A, B, C, and D, respectively. Each subscale contains seven items which are each scored $0-0-1-1$, so that the number of possible scores on each subscale is seven ( 0 to 7$)$, and that on the full questionnaire 28 (0 to 28). The scores on subscales A, B, C, and D were considered non-specific measures of current problems: somatic symptoms, anxiety and insomnia, social dysfunction, and symptoms of depression, respectively. At the same time, scores greater than 4 on the GHQ as a whole were taken to show current non-specific psychiatric morbidity. Both questionnaires were administered to each participant by a trained interviewer immediately before oesophageal manometry in the case of the non-cardiac chest pain patients, or one to two days before the scheduled surgery in the case of the gallstone patients. The interviewer had no knowledge of the reason for referral, and had no clinical information related to the patients. The final psychiatric diagnoses were made by one of the authors (KYH) without knowledge of the manometric results.

Table 2 Percentage of subjects with psychiatric disgnoses by subject group

\begin{tabular}{|c|c|c|c|c|}
\hline & Group I $(n=39)$ & Group II $(n=22)$ & Group III $(n=36)$ & $p$ Value \\
\hline Panic disorder & $15^{\star}$ & 0 & 0 & $<0.01$ \\
\hline Generalised anxiety & 15 & 18 & 3 & NS \\
\hline Phobic disorder & 26 & 14 & 14 & NS \\
\hline $\begin{array}{l}\text { Major depressive } \\
\text { episode }\end{array}$ & $28 \dagger$ & 27 & 8 & NS \\
\hline $\begin{array}{l}\text { Obsessive-compulsive } \\
\text { disorder }\end{array}$ & $21^{\star}$ & 5 & 3 & $<0.03$ \\
\hline Somatisation & 5 & 9 & 0 & NS \\
\hline Any of the above & 51 & 41 & 22 & $<0.04$ \\
\hline
\end{tabular}

STATISTICAL ANALYSIS

Between group differences were assessed using the $\chi^{2}$ or Kruskal-Wallis test, as appropriate. These were followed by pairwise multiple comparisons among the three groups by means of Fisher's exact test and the Mann-Whitney U test, respectively. The results of these comparisons are reported as unadjusted $\mathrm{p}$ values. A Bonferroni correction to allow for the three pairwise comparisons would require the unadjusted $\mathrm{p}$ value to be less than 0.02 before one could claim significance at the 5\% level. Multivariate analysis was performed using logistic regression analysis. The odds ratios (OR) and 95\% confidence intervals (CI) for each significant variable in the final model were calculated from the coefficients in the logistic regression models. All statistics were analysed using the Statistical Package for the Social Sciences for Windows (SPSS, Chicago, Illinois, USA).

\section{Results}

A total of 70 consecutive patients with non-cardiac chest pain was referred for oesophageal manometry and 24 hour $\mathrm{pH}$ monitoring over a period of 27 months. They had previously undergone coronary angiography $(\mathrm{n}=17)$, dobutamine stress echocardiography $(n=8)$, and/or exercise electrocardiogram $(n=45)$. Three patients refused to participate in the questionnaire interview, two were excluded because of language difficulty, and four missed the interview, leaving 61 who completed the questionnaire (response rate $87 \%$ ). Nonrespondents were analysed with reference to sex, age, ethnic group, education level, occupation, marital status, and duration of chest pain, and were found not to differ significantly from those who participated. Among respondents, four had peptic ulcer disease (three with gastric ulcer and one with duodenal ulcer), six had endoscopic oesophagitis (grade 1 in five; grade 2 in one), one had manometric criteria consistent with diffuse oesophageal spasm, and 12 had increased oesophageal acid exposure time (one of whom also had endoscopic oesophagitis; mean oesophageal acid exposure time: $8.5 \%$, $95 \%$ CI 6.3 to 10.7 ). The remaining 39 had no significant endoscopic findings, manometric abnormalities, or pathological reflux.

Over a period of four months in the same period, 52 patients with symptomatic gallstone disease presented to the hospital for elective cholecystectomy. Of these, five refused to participate, four were discharged before they could be approached, four could not be contacted despite several attempts, two were too frail to be interviewed, and one had a language difficulty. The remaining 36 were interviewed (response rate $69 \%$ ). There was no significant difference in sex composition, age, ethnicity, educational level, occupation, marital status, or duration of abdominal pain between those who participated and those who did not.

PATIENT CHARACTERISTICS

The final sample consisted of 39 patients with chest pain and no clinically significant endoscopic abnormality, abnormal motility, or gastro-oesophageal reflux (group I), 22 
Table 3 Scores of psychological morbidity on the 28 item General Health Questionnaire and its subscales by subject group

\begin{tabular}{lllll}
\hline & Group I $(n=39)$ & Group II $(n=22)$ & Group III $(n=36)$ & $p$ Value \\
\hline General Health Questionnaire & $6.1(3.9-8.3)^{\star}$ & $3.6(1.6-5.6)$ & $2.2(0.8-3.6)$ & $<0.005$ \\
$\quad$ Somatic symptoms (A) & $2.4(1.8-3.1)^{\star}$ & $1.5(0.8-2.2)$ & $1.3(0.6-1.9)$ & $<0.02$ \\
Anxiety and insomnia (B) & $1.6(0.9-2.3)^{\star}$ & $1.2(0.5-1.9) \ddagger$ & $0.6(0.03-1.1)$ & $<0.01$ \\
Social dysfunction (C) & $1.2(0.5-1.8) \dagger$ & $0.6(-0.1-1.4)$ & $0.2(0.04-0.4)$ & $<0.05$ \\
Severe depression (D) & $0.9(0.3-1.5) \dagger$ & $0.3(-0.2-0.8)$ & $0.1(-0.1-0.4)$ & NS \\
General Health Questionnaire $>4(\%)$ & $49^{\star}$ & 27 & 14 & $<0.005$ \\
\hline
\end{tabular}

Data are expressed as mean $(95 \% \mathrm{CI})$ unless otherwise stated.

${ }^{\star} \mathrm{p}<0.005 v$ group III.

$t \mathrm{p}<0.05 v$ group III however the difference did not reach the level of significane of $\mathrm{p} \leqslant 0.02$ established by the Bonferroni correction. $\neq \mathrm{p}<0.02 v$ group III.

patients with clinically relevant endoscopic abnormality, abnormal motility, and/or gastrooesophageal reflux (group II), and 36 patients with symptomatic gallstone disease awaiting cholecystectomy (group III).

The three study groups did not differ in terms of age, sex, ethnic background, highest educational level attained, marital status, or manometric findings (table 1). However, there were significant differences with regard to occupation and duration of pain: group I patients had a higher proportion of manual workers and unemployed people compared with group II; group I patients also reported a longer duration of pain compared with the other groups.

PSYCHIATRIC DIAGNOSES

Applying the DSM-111-R criteria and using DIS as the interview method, group I patients were found to have a significantly greater chance of meeting criteria for at least one lifetime psychiatric diagnosis $(51 \%)$ than those in group III $(22 \%, \mathrm{p}<0.02)$ (table 2$)$. The condition that best distinguished the two groups was panic disorder, which was found among 15\% of group I, and none of group III patients $(\mathrm{p}<0.02)$. In addition, obsessive-compulsive disorder $(21 \%$ versus $3 \%, \mathrm{p}<0.02)$ and major depressive episode $(28 \%$ versus $8 \%, \mathrm{p}<0.05)$ were found significantly more frequently in group I than in group III patients; however, the latter difference did not meet our stringent criterion for statistical significance $(p \leqslant 0.02)$ as established by the Bonferroni correction procedure. There were no significant differences between groups II and III or between groups I and II with respect to any of the psychiatric diagnoses. The frequency of psychiatric problems was not related to sex, age, ethnicity, educational level, marital status, occupation, duration of pain, or manometric findings.

\section{PSYCHIATRIC MORBIDITY}

Table 3 summarises the data gained from responses to the GHQ. The overall score and scores on each of its subscales were significantly higher in group I than in group III. The group differences in overall score and scores on its somatic symptoms subscale, as well as its anxiety and insomnia subscale, persisted even after adjustment for multiple comparisons was made. In contrast, group II patients recorded significantly higher scores only on the overall GHQ and its anxiety and insomnia subscale when compared with group III patients. After correcting for multiple comparisons, only the anxiety and insomnia subscale reliably differentiated between groups II and III. Group I and II patients did not differ in any of the scores. The data from the GHQ were also examined in terms of the proportion of patients who scored above the cut off point (that is, scores above 4). Forty nine per cent of group I patients scored high enough to have possible non-psychotic psychiatric disturbance, compared with $14 \%$ of group III patients $(\mathrm{p}<0.002)$, and $27 \%$ of group II patients (NS). Scores on the GHQ and its subscales were not influenced by sociodemographic variables such as sex, age, ethnic background, educational level, marital status, occupation, duration of pain, or manometric findings.

DISCRIMINANT ANALYSES

To examine the interaction between groups I and III further, we performed a stepwise multiple regression analysis. With groups I versus III as the dependent variable, tests were run on a model utilising panic disorder, obsessivecompulsive disorder, major depressive episode, GHQ score greater than 4, and duration of pain - all of which were univariately significant variables. The analysis showed a significant and independent contribution from GHQ score greater than 4 (OR 5.24, 95\% CI 1.42 to 19.34 ) and duration of pain (OR 1.12, 95\% CI 1.04 to 1.22 ) but no effect of panic disorder, obsessive-compulsive disorder, or major depressive episode.

\section{Discussion}

The present study shows that patients with non-cardiac chest pain and no clinically significant upper gastrointestinal disease had a higher prevalence of lifetime psychiatric diagnoses than those with symptomatic gallstone disease. The diagnoses of panic disorder, obsessive-compulsive disorder, and major depressive episode were responsible for the overall increase in this group. In addition, these subjects produced higher scores indicative of current psychiatric morbidity compared with the patients with gallstones. In contrast, patients with non-cardiac chest pain and no significant upper gastrointestinal disease were similar to patients with gallstones in the frequency of lifetime psychiatric diagnoses as well as the degree of psychiatric comorbidity. These results suggest that psychological factors may be relevant in the pathogenesis of chest pain in patients who have no obvious cardiac or oesophagogastric disease. On the other hand, patients with non-cardiac chest pain who had 
an underlying oesophagogastric disorder causing the symptom were not at increased risk of psychopathology compared with patients having pain due to gallstone disease.

The strengths of this study include the use of blinded evaluations and validated psychiatric instruments, an adequate sample size with no statistical significance between subjects who participated in the study and those who did not, and stringent criteria using the Bonferroni method for accepting relations as statistically significant. The duration of pain among patients with non-cardiac non-oesophagogastric pain was greater, compared with the other two groups. This, however, cannot possibly account for the increased prevalence of psychiatric diagnoses and psychiatric comorbidity among these patients, as no relation was found between pain duration and any of the psychiatric variables. Furthermore, psychiatric comorbidity remained a highly significant risk marker of non-cardiac and non-oesophagogastric chest pain as against gallstone pain, even after taking into account the duration of pain in the logistic model.

All the patients with non-cardiac chest pain underwent psychological assessment after upper gastrointestinal endoscopy. It could be argued that knowledge of the endoscopic findings might have influenced their responses to the questionnaire. For example, patients with abnormal endoscopy might have been reassured by the procedure, while those with normal endoscopy might have remained psychologically disturbed because of uncertainty concerning the cause of their pain. However, this possibility seems unlikely, as the results did not differ even after excluding patients with peptic ulcer disease and endoscopic oesophagitis from the analysis.

Oesophageal provocation tests such as the acid perfusion test, and qualitative analysis of $\mathrm{pH}$ measurements such as the assessment of symptoms and reflux correlation are frequently used in the investigation of patients with non-cardiac chest pain. While these were performed in our patients, the results were not utilised for the present study for the following reason. The tests depend on subjective reporting of pain, which can be modified by factors such as anxiety, the personality of the subject, and a desire to please the investigator. Subjects with panic disorder and/or obsessivecompulsive disorder might thus be more likely to report pain on these tests than those without. Including such patients in the group with clinically significant oesophageal disorders might erroneously increase the proportion of subjects with psychological dysfunction and falsely nullify the conclusions reached by the present study.

It is widely recognised that oesophageal dysfunction, particularly gastro-oesophageal reflux disease, is a common cause of non-cardiac chest pain in Western populations. ${ }^{23}$ The frequency of endoscopic oesophagitis in patients presenting for endoscopy, ${ }^{16}$ symptomatic gastro-oesophageal reflux in pregnant women (unpublished data, KYH, 1997), and reflux type symptoms in the community generally ${ }^{17}$ is lower among Singaporeans than their Western counterparts. Despite this, gastro-oesophageal reflux disease is the most common cause of non-cardiac chest pain in the East, ${ }^{1}$ as in the West. The cause of chest pain that is neither cardiac nor oesophageal in origin is unknown, but previous studies have suggested that psychological factors might be responsible. ${ }^{5} \mathrm{We}$ have extended these observations by dividing non-cardiac chest pain patients into those with clinically significant oesophageal or gastric pathology and those without. The results show that psychiatric factors were relevant only in those for whom oesophageal or gastric disease had been excluded. Klimes et al have found psychological treatment to be beneficial for patients with non-cardiac chest pain. ${ }^{4}$ Our results suggest that psychological treatment may be appropriate only for those who have no structural, reflux related, or clinically significant motility disorder in the upper gastrointestinal tract.

An association of psychiatric factors with non-specific oesophageal motility disorders has been reported previously. ${ }^{67}$ Clouse and Lustman found a higher prevalence of psychiatric diagnoses among patients with nonspecific oesophageal motility disorders when compared with those having specific oesophageal motility disorders like achalasia, or having normal manometry. ${ }^{6}$ They suggested that psychiatric illness might be relevant in the generation of these non-specific manometric features. A similar conclusion was reached by Richter et $a l,{ }^{7}$ who studied somatic anxiety scores in patients with nutcracker oesophagus. Such conclusions may, however, be biased in two ways. Firstly, the functional significance of non-specific oesophageal motility disorders, including nutcracker oesophagus, has not been established. ${ }^{10}$ Secondly, clinically significant oesophageal or cardiac pathology was present in a sizeable proportion of controls in the two studies. Thus, the high levels of psychological distress seen in patients with non-specific oesophageal motility disorder may have been related to the absence of organic oesophageal or cardiac pathology rather than to the incidental presence of nonspecific motor disturbances. We have carefully designed the present study to reveal structural, motility, and reflux related disorders of the upper gastrointestinal tract by a standardised protocol. It was found that psychological factors were related to the absence of clinically significant lesions in the heart or upper gastrointestinal tract, but not to the presence of non-specific oesophageal motility disturbances.

It is interesting to note that current psychological morbidity as indicated by the GHQ score was present in less than half the cases, even among those with non-cardiac and oesophagogastric chest pain. There remains therefore a proportion of patients with no definite aetiology to explain their chest pain. Further studies on the role of oesophageal and cardiac visceral hypersensitivity would be helpful for these individuals. 
The prevalence of current psychiatric morbidity and lifetime psychiatric disorder in our sample of Asian patients was comparable with that reported in studies of Western patients. ${ }^{56}$ The range of psychiatric abnormalities we observed was also similar to those reported in previous studies, ${ }^{5618}$ with panic disorder emerging as the most common manifestation. These data seem to run counter to the generally held view that somatic presentation of psychiatric disorders occurs more frequently among Oriental cultures than Western ones. ${ }^{19}$ However, in support of our findings, a recent international primary care study found somatic symptoms to be strongly associated with psychological distress across all cultures and all levels of economic development. ${ }^{20}$

This work was supported by Academic Research Grant RP900364 from the National University of Singapore. The authors wish to thank $\mathrm{Mr} \mathrm{CO} \mathrm{Ho}$ for technical assistance and Dr KA Gwee for statistical advice.

1 Ho KY, Kang JY, Ng WL. Is the oesophagus a common origin of non-cardiac chest pain in Singapore [abstract]? $f$ Gastroenterol Hepatol 1995;10(suppl 3):A75.

2 Ghillebert G, Janssens J, Vantrappen G, et al. Ambulatory 24 hour intra-esophageal $\mathrm{pH}$ and pressure recording versus provocation tests in the diagnosis of chest pain of provocation tests in the diagnosis

3 Hewson EG, Dalton CB, Richter JE. Comparison of esophageal manometry, provocative testing and ambulatory monitoring in patients with unexplained chest pain. tory monitoring in patients

4 Klimes I, Mayou RA, Pearce MJ, et al. Psychological treatment for atypical non-cardiac chest pain: a controlled evaluation. Psychol Med 1990;20:605-11.

5 Katon W, Hall ML, Russo J, et al. Chest pain: relationship of psychiatric illness to coronary arteriography results. $A m \mathcal{F}$ Med 1988;84:1-9.
6 Clouse RE, Lustman PJ. Psychiatric illness and contraction abnormalities of the esophagus. N Engl f Med 1983;309: abnormalitie

7 Richter JE, Obrecht WF, Bradley LA, et al. Psychological comparison of patients with nutcracker esophagus and irritable bowel syndrome. Dig Dis Sci 1986;31:131-8.

8 Yeoh KG, Ho KY, Guan R, et al. How does chili cause upper gastrointestinal symptoms? A correlation study with esophageal mucosal sensitivity and esophageal motility. $f$ Clin Gastroenterol 1995;21:87-90.

9 Katz PO, Dalton CB, Richter JE, et al. Esophageal testing of patients with noncardiac chest pain or dysphagia: results of three years' experience with 1161 patients. Ann Intern Med 1987;106:593-7.

10 Kahrilas PJ, Clouse RE, Hogan WJ. American Gastroenterological Association technical review on the clinical use of esophageal manometry. Gastroenterology 1994;107: $1865-84$.

11 Robins LN, Helzer JE, Croughan J, et al. National Institute of Mental Health Diagnostic Interview Schedule: its history, characteristics, and validity. Arch Gen Psychiatry 1981;38:381-9.

12 Goldberg DP, Hillier V. A scaled version of the General Health Questionnaire. Psychol Med 1979;9:139-45.

13 American Psychiatric Association, Committee on Statistics. Diagnostic and statistical manual of mental disorders, revised third edition. Washington, DC: American Psychiatric Association, 1987.

14 Katon WJ, Sullivan MD. Depression and chronic medical illness. F Clin Psychiatry 1990;51(suppl):3-11.

15 Stansfeld SA, Smith GD, Marmot M. Association between physical and psychological morbidity in the Whitehall II physical and psychological morbidity in

16 Kang JY, Tay HH, Yap I, et al. Low frequency of endoscopic esophagitis in Asian patients. F Clin Gastroenterol 1993;16: $70-3$

17 Ho KY, Kang JY, Seow A. Prevalence of chronic gastrointestinal symptoms among Chinese, Malay and Indian adults in Singapore [abstract]. $\mathcal{F}$ Gastroenterol Hepatol 1995;10(suppl 3):A98.

18 Beitman BD. Panic disorder in patients with angiographically normal coronary arteries. Am $\mathcal{F}$ Med 1992;92 (suppl $5 \mathrm{~A}): 33 \mathrm{~S}-40 \mathrm{~S}$.

19 Kleinman AM. Depression, somatization and the "new cross-cultural psychiatry". Soc Sci Med 1977;11:3-10.

20 Simon G, Gater R, Kisely S, et al. Somatic symptoms of distress: an international primary care study. Psychosom Med 1996;58:481-8. 